\title{
Achievable Rate Region of CSMA Schedulers in Wireless Networks with Primary Interference Constraints
}

\author{
Peter Marbach, Atilla Eryilmaz, and Asuman Ozdaglar
}

\begin{abstract}
We consider Carrier Sense Multiple Access (CSMA) schedulers for wireless networks. For networks where all nodes are within transmission range of each other, it has been shown that such schedulers achieve the network capacity in the limiting region of large networks with a small sensing delay. However the design and analysis of CSMA schedulers for general networks has been an open problem due to the complexity of the interaction among coupled interference constraints. For networks with primary interference constraints, we introduce a tractable analysis of such CSMA schedulers based on a fixed point approximation. We then use the approximation to characterize the achievable rate region of static CSMA schedulers. We show that the approximation is asymptotically accurate for the limiting regime of large networks with a small sensing delay, and that in this case the achievable rate region of CSMA converges to the capacity region.
\end{abstract}

\section{INTRODUCTION}

Recently, there has been a growing interest in the development of distributed transmission policies for interferencelimited wireless networks. A key objective of these distributed policies is to achieve any throughput within the capacity region ${ }^{1}$ of the network. In this paper we focus on Carrier Sense Multiple Access (CSMA) schedulers where nodes sense whether the channel is idle before making an attempt to transmit a packet. For single-hop networks where all nodes are within transmission range of each other, it is well-known that CSMA schedulers achieve network capacity in the limiting regime of large networks with a small sensing time [2]. However the analysis of CSMA schedulers for general networks has been an open problem due to the complexity of the interaction among coupled interference constraints. In this paper, we provide an analysis of CSMA schedulers for networks with primary interference constraints.

The main contributions of the paper are as follows. We provide a fixed point approximation, called the CSMA fixed point, to characterize the service rates of CSMA schedulers in networks with primary interference constraints, and show that (under some assumptions) the fixed point approximation

P. Marbach is with the Department of Computer Science at the University of Toronto, Toronto, Canada. Email: marbachecs . toronto.edu.

A. Eryilmaz is with the Department of Electrical and Computer Engineering at the Ohio State University, Columbus, USA. Email: eryilmaz.2@osu.edu.

A. Ozdaglar is with the Department of Electrical Engineering and Computer Science at the Massachussettes Institute of Technology, Cambridge, USA. Email: asuman@mit.edu.

The work of the second and third authors was supported by the ControlBased Mobile Ad-Hoc Networking (CBMANET) Program under DARPA subcontract no. 060786 .

${ }^{1}$ The capacity region contains the set of arrival rates that are achievable by some feasible policy. A more rigorous definition will be provided later. is asymptotically accurate for large networks with a small sensing time. Using the fixed point approximation, we characterize the achievable rate region of the static CSMA schedulers for networks with primary interference constraints. We show that the achievable rate region converges to the capacity region for large networks with a small a sensing time.

Most recent work in this area focused on distributed mechanisms that can implement the throughput-optimal (or max-weight) policies (see for example [3], [7], [8], [10], [12]) that has been proposed in the seminal work of Tassiulas and Ephremides [11]. In view of their low-complexity nature, random access schemes provide an attractive alternative class of distributed transmission strategies; however, the current understanding of such schemes is still limited. Random access protocols have been analyzed in [13] by Durvy and Thiran using a packing approach, and in [14] by Bordenave, McDonald, and Proutiere, using a mean-field analysis approach. Related to [14], in this paper we analyze CSMA random access schemes using the CSMA fixed point approximation.

The paper is organized as follows. We describe the wireless network and the static CSMA schedulers that we consider for our analysis in Section II. In Section III, we review well-know results for networks where all nodes share a single communication channel. Section IV presents the CSMA fixed point formulation that we use to approximate the performance of static CSMA schedulers. We show in Section IV that there always exists a unique CSMA fixed point and hence our fixed point approximation is welldefined. In Section V, we show that the fixed point approximation is asymptotically accurate for large networks with a small sensing delay. Section VI uses the CSMA fixed point approximation to characterize the achievable rate region of static CSMA schedulers. Finally, in Section VII, we show that the achievable rate region asymptotically converges to the capacity region for large networks with a small sensing time. Due to space constraints, we state our results without proofs.

\section{Problem Formulation}

Consider a wireless network consisting of $N$ nodes and $L$ directed links. A link $(i, j)$ is given by a node pair $i$ and $j$, where $i$ is the sending and $j$ is the receiving node. Let $\mathscr{L}$ be the set of all links in the network. Let $S_{i}$ be the set of all nodes $j$ such that there exists a link from node $i$ to node $j$, and let $R_{i}$ be the set of all nodes $j$ for which there exists a link from node $j$ to node $i$. Let $\mathscr{N}_{i}=S_{i} \cup R_{i}$ denote the neighbourhood of node $i$. Furthermore let $\mathscr{L}_{i}$ be the set of 
links originating at node $i$, i.e. we have that

$$
\mathscr{L}_{i}=\{(k, j) \in \mathscr{L} \mid k=i\} .
$$

In the following we assume that $S_{i}=R_{i}, i=1, \ldots, N$, i.e. all nodes have the same transmission range.

For our analysis, we focus on networks under the primary interference, or node exclusive interference, model as given below (see also [8], [12]).

Definition 1 (Primary Interference Model): An attempt by node $i$ to capture link $(i, j)$ is successful if it does not overlap with any attempt by another node to capture a link $(k, l)$ which has a node in common with link $(i, j)$.

This interference model applies, for example, to wireless systems where multiple frequencies/codes are available (using FDMA or CDMA) to avoid interference, but each node has only a single transceiver and hence can only send to or receive from one other node at any time (see [5] for additional discussion).

\section{A. Static CSMA Schedulers}

We consider a Carrier Sense Multiple Access (CSMA) mechanism to schedule the times when nodes try to capture a link that is given as follows.

Before trying to capture a link, nodes sense whether the link is idle. More precisely, we say that a node $i$ is idle if it currently does not capture any link $(i, j), j \in R_{i}$, nor does any node $j \in S_{i}$ capture the link $(j, i)$. We say that the link $(i, j)$ is idle if both node $i$ and node $j$ are idle. Nodes only try to capture links that have been idle for $\beta$ time units. We refer to $\beta$ as the sensing time (or delay), and define an idle slot for link $(i, j)$ as the event that link $(i, j)$ has been idle for $\beta$ time units. In addition, we define the end of an idle period at link $(i, j)$ as the event that node $i$ and $j$ have been jointly idle for exactly $k$ idle slots, $k \in\{1,2, \ldots\}$.

Definition 2 (Static CSMA Policy): A static CSMA policy is given by a vector $\mathbf{p}=\left(p_{(i, j)}\right)_{(i, j) \in \mathscr{L}} \in[0,1]^{L}$. At a given time $t \geq 0$, let $\mathscr{L}_{i}(t)$ be the set of links in $\mathscr{L}_{i}$ for which an idle period ends at time $t$. Then node $i$ will mark link $(i, j) \in \mathscr{L}_{i}(t)$ at time $t$ with probability $p_{(i, j)}$, independently of all other attempts by any node in the network. If at time $t$, node $i$ marks a single link $(i, j) \in \mathscr{L}_{i}(t)$, then it will make an attempt at time $t$ to capture that link. If node $i$ marks two, or more, links in the set $\mathscr{L}_{i}(t)$ at time $t$, then it will choose an arbitrary link among the marked links, say $(i, j)$, and will make an attempt at time $t$ to capture the link $(i, j)$.

If a node tries to capture a link, it will hold (occupy) the link for one packet transmission duration. In the following, we assume that the transmission time of each packet is equal to 1 time unit. If a node does not make an attempt to capture a link, then it will wait until the next time that an idle period of a link $(i, j), j \in \mathscr{N}_{i}$, ends.

To simplify the analysis, we assume in the rest of the paper that the propagation delay between a node $i$ and a node $j \in \mathscr{N}_{i}, i \neq j$ (i.e. the time it takes a node $i$ to detect that node $j$ stopped/started transmitting) is equal to the sensing time $\beta$. Furthermore, we assume that all nodes are initially idle and start sensing at time $t=0$. The analysis can be extended (but becomes more involved) to the case where the propagation delay between neighbouring nodes is upper-bounded by $\beta$ and the initial sensing times are not aligned.

\section{B. Performance Measures}

We define the service rate of a static CSMA policy for a link $(i, j) \in \mathscr{L}$ as follows.

Definition 3 (Service Rate): The service rate $T_{(i, j)}(\mathbf{p})$ of a static CSMA policy $\mathbf{p}$ for link $(i, j) \in \mathscr{L}$ is the fraction of time node $i$ successfully captures link $(i, j)$ under $\mathbf{p}$. $\diamond$ Note that the service rate $T_{(i, j)}(\mathbf{p})$ of the link $(i, j)$ is equal to the throughput for the link $(i, j)$ under the static CSMA policy $\mathbf{p}$ for the case where all links are saturated, i.e. there is always a packet to be sent for all links $(i, j) \in \mathscr{L}$. Using the service rates $T_{(i, j)}(\mathbf{p}),(i, j) \in \mathscr{L}$, we next define the achievable rate region of static CSMA policies.

Let $\mathscr{R}$ be a given set of routes for the above wireless network, where route $r \in \mathscr{R}$ is characterized by the links that it traverses. Let $\lambda=\left\{\lambda_{r}\right\}_{r \in \mathscr{R}} \in \mathbb{R}_{+}^{|\mathscr{R}|}$ be the mean packet arrival rate vector where $\lambda_{r}$ is the mean packet arrival rate for route $r$. Given a rate vector $\lambda$, let

$$
\lambda_{(i, j)}=\sum_{r:(i, j) \in r} \lambda_{r}, \quad(i, j) \in \mathscr{L},
$$

be the total mean packet arrival rate over all routes $r \in \mathscr{R}$ that traverse link $(i, j)$, and let

$$
\Lambda_{i}=\sum_{j \in \mathscr{N}_{i}}\left[\lambda_{(i, j)}+\lambda_{(j, i)}\right], \quad i=1, . ., N,
$$

be the total mean packet arrival rate over all routes $r \in \mathscr{R}$ that pass through node $i$.

We assume that the incoming packets for each link $(i, j)$ are stored in a separate input-queue of infinite size.

Definition 4 (Stability): Consider an arrival rate vector $\lambda=\left\{\lambda_{r}\right\}_{r \in \mathscr{R}}$ and a static CSMA policy $\mathbf{p}$ with service rates $T_{(i, j)}(\mathbf{p}),(i, j) \in \mathscr{L}$. We say that $\mathbf{p}$ stabilizes the network if

$$
\lambda_{(i, j)}<T_{(i, j)}(\mathbf{p}), \quad(i, j) \in \mathscr{L},
$$

i.e. the arrival rate is strictly less than the service rate on all links $(i, j) \in \mathscr{L}$.

The achievable rate region of static CSMA policies is then given as follows.

Definition 5 (Achievable Rate Region): The achievable rate region of static CSMA policies is the set of arrival rate vectors $\lambda=\left\{\lambda_{r}\right\}_{r \in \mathscr{R}}$ for which there exists a static CSMA policy that stabilizes the network.

In the following we characterize the service rates of a given static CSMA policy, as well as the achievable rate region of static CSMA policies.

\section{Single-HoP NETWORKS}

Before we present our analysis of static CSMA policies for general networks, we review in this section well-known results on CSMA schedulers for networks where $N$ nodes share a single communication channel [2]. In this case, an attempt by a node to capture the channel after an idle period is only successful if it does not overlap with an attempt by 
any other node. A static CSMA policy is then given by the vector $\mathbf{p}=\left(p_{1}, \ldots, p_{N}\right) \in[0,1]^{N}$ where $p_{n}$ is the probability that node $n$ makes an attempt to capture the channel. Under a given static CSMA policy $\mathbf{p}$, the service rate $T_{n}(\mathbf{p})$ of node $n$ is the fraction time node $n$ successfully captures the channel under $\mathbf{p}$. The network service rate $T(\mathbf{p})$ is given by

$$
T(\mathbf{p})=\sum_{n=1}^{N} T_{n}(\mathbf{p}) .
$$

For a given static CSMA scheduling policy $\mathbf{p}=$ $\left(p_{1}, \ldots, p_{N}\right)$, let $G(\mathbf{p})=\sum_{n=1}^{N} p_{n}$ denote the rate (also called the offered load) with which nodes make attempts to capture the channel at the end of an idle period. For a given static CSMA policy $\mathbf{p}$, the following approximation for the network service rate is well-known (see for example [2]),

$$
\tau(G(\mathbf{p}))=\frac{G(\mathbf{p}) e^{-G(\mathbf{p})}}{\beta+1-e^{-G(\mathbf{p})}}
$$

For $\beta>0$, one can show that (see for example [2])

$$
\tau(G)<1, \quad G \geq 0,
$$

and for $G^{+}(\beta)=\sqrt{2 \beta}, \beta>0$, we have that

$$
\lim _{\beta \downarrow 0} \tau\left(G^{+}(\beta)\right)=1 .
$$

Using Eq. (3), the service rate $\tau_{n}(\mathbf{p})$ of node $n$ under a given static CSMA policy $\mathbf{p}$ can be approximated by

$$
\tau_{n}(\mathbf{p})=\frac{p_{n} e^{-G(\mathbf{p})}}{1+\beta-e^{-G(\mathbf{p})}}, \quad n=1, \ldots, N .
$$

In the above expression, $p_{n}$ is the probability that node $n$ tries to capture the channel after an idle period and $e^{-G(\mathbf{p})}$ characterizes the probability that this attempt is successful, i.e. the attempt does not collide with an attempt by any other node.

Similarly, the fraction of time that the channel is idle can be approximated by

$$
\rho(\mathbf{p})=\rho(G(\mathbf{p}))=\frac{\beta}{\beta+1-e^{-G(\mathbf{p})}},
$$

where we have that $\lim _{\beta \downarrow 0} \rho\left(G^{+}(\beta)\right)=0$.

Eq. (3)-(7) are obtained by approximating the number of nodes that make an attempt to capture the channel after an idle period by a Poisson distribution with mean $G(\mathbf{p})$. This approximation is asymptotically accurate for large networks where each node makes an the attempt to capture the channel with a small probability. More precisely, consider a sequence of networks with $N$ nodes and let $\left\{\mathbf{p}^{(N)}\right\}_{N \geq 1}, \mathbf{p}^{(N)} \in[0,1]^{N}$, be a sequence of CSMA policies such that

$$
\sum_{n=1}^{N} p_{n}^{(N)}=G, \quad N \geq 1
$$

for some constant $G \geq 0$, and $\lim _{N \rightarrow \infty} p_{n}^{(N)}=0, \forall n$. Under the above scaling, the offered load stays constant, and equal to $G$, as the number of nodes $N$ increases.

Let $T\left(\mathbf{p}^{(N)}\right)$ be the network service rate for the network with $N$ nodes, and let $\bar{\rho}\left(\mathbf{p}^{(N)}\right)$ be the fraction of time that the channel is idle. For the above scaling, it is then well-known (see for example [15]) that the number of nodes that make an attempt to capture the channel after an idle period weakly converges to a Poisson random variable with mean $G$. Using this result, it then follows that

$\lim _{N \rightarrow \infty}\left|T\left(\mathbf{p}^{(N)}\right)-\tau(G)\right|=0$ and $\lim _{N \rightarrow \infty}\left|\bar{\rho}\left(\mathbf{p}^{(N)}\right)-\rho(G)\right|=0$, i.e. the approximations given by Eq. (3) and Eq. (7) are asymptotically accurate.

\section{CSMA FIXED POINT ANALYSIS}

In this section, we approximate the service rates of a static CSMA policy $\mathbf{p}$ for a general network using a fixed point analysis. In the Section V, we show that this approximation is asymptotically accurate for large networks with a small sensing time.

\section{A. Fixed Point Formulation}

Given a static CSMA policy $\mathbf{p}$, we approximate the fraction of time $\rho_{i}(\mathbf{p})$ that node $i$ is idle under $\mathbf{p}$ by the following fixed point equation,

$$
\rho_{i}(\mathbf{p})=\frac{\beta}{\left(\beta+1-e^{-G_{i}(\mathbf{p})}\right)}, \quad i=1, \ldots, N,
$$

where $G_{i}(\mathbf{p})$ is given by

$$
G_{i}(\mathbf{p})=\sum_{j \in \mathscr{N}_{i}}\left[p_{(i, j)}+p_{(j, i)}\right] \rho_{j}(\mathbf{p}), \quad i=1, \ldots, N
$$

Let $G(\mathbf{p})=\left(G_{1}(\mathbf{p}), \cdots, G_{N}(\mathbf{p})\right)$. We refer to Eq. (8) as the CSMA fixed point equation and to $\rho(\mathbf{p})=$ $\left(\rho_{1}(\mathbf{p}), \cdots, \rho_{N}(\mathbf{p})\right)$, as well as $G(\mathbf{p})=\left(G_{1}(\mathbf{p}), \ldots, G_{N}(\mathbf{p})\right)$, as the CSMA fixed point.

The intuition behind the CSMA fixed point can be explained as follows. Suppose that the fraction of time that node $i$ is idle under the static CSMA policy $\mathbf{p}$ is equal to $\rho_{i}(\mathbf{p})$, and suppose that the times when node $i$ is idle are independent of the processes at all other nodes. If node $i$ has been idle for $\beta$ time units then the probability that node $i$ makes an attempt to capture the $\operatorname{link}(i, j), j \in \mathscr{N}_{i}$, is equal to $p_{(i, j)} \rho_{j}(\mathbf{p})$, and the probability that node $j \in \mathscr{N}_{i}$ makes an attempt to capture the link $(j, i)$ is $p_{(j, i)} \rho_{j}(\mathbf{p})$. Hence, the rate at which node $i$ receives or makes attempts to capture a link after it has been idle for $\beta$ time units is given by Eq. (9). Using Eq. (7) of Section III, the fraction of time that node $i$ is idle under $\mathbf{p}$ can be approximated by Eq. (8).

Let

$$
G_{i}^{R}(\mathbf{p})=\sum_{j \in \mathscr{N}_{i}} p_{(j, i)} \rho_{j}(\mathbf{p})
$$

Using the above CSMA fixed point formulation, we approximate the service rate for link $(i, j)$ under a static CSMA policy $\mathbf{p}$ by

$$
\tau_{(i, j)}(\mathbf{p})=\frac{p_{(i, j)} \rho_{j}(\mathbf{p}) e^{-\left(G_{i}^{R}(\mathbf{p})+G_{j}(\mathbf{p})\right)}}{1+\beta-e^{-G_{i}(\mathbf{p})}}
$$

Note that the above equation is similar to Eq. (6) where $p_{(i, j)} \rho_{j}(\mathbf{p})$ captures the probability that node $i$ makes an attempt to capture link $(i, j)$ if it has been idle for $\beta$ time 
units, and $\exp \left[-\left(G_{i}^{R}(\mathbf{p})+G_{j}(\mathbf{p})\right)\right]$ is the probability that this attempt is successful, i.e. the attempt does not overlap with an attempt by any other node to capture a link that has an endpoint in common with link $(i, j)$. Note that

$$
\tau_{(i, j)}(\mathbf{p}) \geq \frac{p_{(i, j)} \beta e^{-\left(G_{i}(\mathbf{p})+G_{j}(\mathbf{p})\right)}}{\left(1+\beta-e^{-G_{i}(\mathbf{p})}\right)\left(1+\beta-e^{-G_{j}(\mathbf{p})}\right)}
$$

as $G_{i}(\mathbf{p}) \geq G_{i}^{R}(\mathbf{p})$.

In the next subsection we show that there always exists a unique CSMA fixed point, and hence the CSMA fixed point approximation (8) is well-defined.

\section{B. Existence of a Unique Fixed Point}

The next proposition shows the existence of a CSMA fixed point for all $\mathbf{p}$.

Lemma 1: For every static CSMA policy $\mathbf{p}$, there exists a CSMA fixed point $\rho(\mathbf{p})$.

The proof for Lemma 1 uses the continuity properties of the fixed point equation given Eq. (8), and is a straightforward application of the Brouwer's fixed point theorem.

The next lemma states that for the CSMA policy $\overline{\mathbf{p}}$ with $\bar{p}_{(i, j)}=0,(i, j) \in \mathscr{L}$, there exists a single CSMA fixed point.

Lemma 2: Consider the CSMA policy $\overline{\mathbf{p}}$ with $\bar{p}_{(i, j)}=0$, $(i, j) \in \mathscr{L}$. Then for any $\beta>0$ the unique CSMA fixed point $\rho(\overline{\mathbf{p}})$ is given by $\rho_{i}(\overline{\mathbf{p}})=1$ and $G_{i}(\overline{\mathbf{p}})=0$, for $i=1, \ldots, N$.

Thes result of Lemma 2 is obtained by applying Eq. (8) and (9) to the CSMA policy $\overline{\mathbf{p}}$ with $\bar{p}_{(i, j)}=0$.

We next study the continuity properties of $G(\mathbf{p})$. To do this, we consider the mapping

$$
f_{i}(G, \mathbf{p})=G_{i}-\sum_{j \in \mathscr{N}_{i}} \frac{\beta\left[p_{(i, j)}+p_{(j, i)}\right]}{\left(1+\beta-e^{-G_{j}}\right)}, \quad i=1, \ldots, N .
$$

Note that the mapping $f(G, \mathbf{p})=\left[f_{i}(G, \mathbf{p})\right]_{i=1, \ldots, N}$ is continuous and we have that $f(G(\mathbf{p}), \mathbf{p})=\mathbf{0}$. We then obtain the following result.

Proposition 1: The correspondence $G:[0,1]^{L} \mapsto \mathbb{R}_{+}^{N}$ is upper-semicontinuous; i.e., $G(\mathbf{p})$ has a closed graph.

Using the mapping $f(G, \mathbf{p})=\left[f_{i}(G, \mathbf{p})\right]_{i=1, \ldots, N}$ given by Eq. (12), we obtain the next proposition which establishes the local uniqueness of the correspondence $G(\mathbf{p})$.

Proposition 2: For all static CSMA policies $\overline{\mathbf{p}}$ and all CSMA fixed points $\bar{G} \in G(\overline{\mathbf{p}})$, there exist neighbourhoods $U \subset \mathbb{R}_{+}^{N}$ of $\bar{G}$ and $V \subset[0,1]^{L}$ of $\overline{\mathbf{p}}$ such that for each $\mathbf{p} \in V$ the equation $f(G, \mathbf{p})=0$ has a unique solution $G \in U$. Moreover, this solution can be given by a function $G=\phi(\mathbf{p})$ where $\phi$ is continuously differentiable on $V$.

Proposition 2 can be proved using the implicit function theorem [1]. Combining Lemma 2 with Propositions 1 and 2 , we obtain the following result.

Theorem 1: For every static CSMA policy $\mathbf{p}$, there exists a unique CSMA fixed point $\rho(\mathbf{p})$.

The uniqueness result of Theorem 1 combined with the upper-semicontinuity of Proposition 1 directly implies the continuity of $G(\mathbf{p})$, and hence of $\rho(\mathbf{p})$. This is stated in the following corollary.

Corollary 1: The fixed point $\rho(\mathbf{p})$ is continuous in $\mathbf{p}$.

\section{Large Networks with a Small Sensing Time}

In this section, we study the behavior of the CSMA fixed point under the limiting regime where the number of nodes $N$ increases to infinity and the sensing time $\beta$ decreases to zero. For this case, we show that the CSMA fixed point description of the operating point is asymptotically accurate. This result states that for large networks with a small sensing time $\beta$, the CSMA fixed point approximates well the actual performance of the static CSMA policy.

Consider a sequence of networks for which the number of nodes $N$ increases to infinity. Let $\mathscr{L}^{(N)}$ be the set of all links in the network with $N$ nodes, and let $\mathscr{N}_{i}^{(N)}$ be the set of neighbours of node $i$. As the network size increases, we assume that the sensing time decreases as follows.

Assumption 1: The sensing time $\beta^{(N)}$ for the network with $N$ nodes given by

$$
\beta^{(N)}=\frac{1}{K^{(N)}}
$$

where $K^{(N)}$ is a positive integer such that

$$
\lim _{N \rightarrow \infty} \frac{N}{K^{(N)}}=0 .
$$

For the above scaling, consider a sequence of static CSMA policies $\left\{\mathbf{p}^{(N)}\right\}_{N \geq 1}$, where $\mathbf{p}^{(N)}$ defines a static CSMA policies for the network with $N$ nodes. We make the following assumptions for the sequence $\left\{\mathbf{p}^{(N)}\right\}_{N \geq 1}$.

Assumption 2: For the sequences $\left\{\mathbf{p}^{(N)}\right\}_{N \geq 1}$ and $\left\{\beta^{(N)}\right\}_{N \geq 1}$ the following is true.

(a) For $p_{\text {max }}^{(N)}=\max _{(i, j) \in \mathscr{L}^{(N)}} p_{(i, j)}^{(N)}$ we have that

$$
\lim _{N \rightarrow \infty} \frac{p_{\max }^{(N)}}{\beta^{(N)}}=0 .
$$

(b) There exists a constant $\chi$ and an integer $N_{0}$ such that for all $N \geq N_{0}$ we have that

$$
\sum_{j \in \mathscr{N}_{i}^{(N)}}\left[p_{(i, j)}^{(N)}+p_{(j, i)}^{(N)}\right] \leq \chi \beta^{(N)}, \quad i=1, \ldots, N .
$$

Condition (a) implies that the attempt probability of each link becomes small as $N$ becomes large, and that the sensing delay does not decrease too fast as $N$ increases. Condition (b) implies that the total rate with which links that originate or end at a given node $i$ are captured, is upper-bounded by $\chi$.

For the above scaling, let $\rho^{(N)}\left(\mathbf{p}^{(N)}\right)=$ $\left(\rho_{1}^{(N)}\left(\mathbf{p}^{(N)}\right), \ldots, \rho_{N}^{(N)}\left(\mathbf{p}^{(N)}\right)\right)$ be the CSMA fixed point for the network of size $N$, and let $\bar{\rho}_{i}^{(N)}\left(\mathbf{p}^{(N)}\right)$ be the actual fraction of time that node $i$ is idle. Furthermore, let

$$
\delta_{\rho}^{(N)}=\max _{i=1, \ldots, N}\left|\rho_{i}^{(N)}\left(\mathbf{p}^{(N)}\right)-\bar{\rho}_{i}^{(N)}\left(\mathbf{p}^{(N)}\right)\right|
$$

be the maximum approximation error of the CSMA fixed point. Similarly, let

$$
\delta_{\tau}^{(N)}=\max _{(i, j) \in \mathscr{L}^{(N)}}\left|1-\frac{\tau_{(i, j)}^{(N)}\left(\mathbf{p}^{(N)}\right)}{T_{(i, j)}^{(N)}\left(\mathbf{p}^{(N)}\right)}\right|
$$


be the maximum relative approximation error of the link service rates under the CSMA fixed point. We following result states that in the limit as $N$ approaches infinity the CSMA fixed point approximation becomes asymptotically accurate.

Proposition 3: For the above defined scaling we have that

$$
\lim _{N \rightarrow \infty} \delta_{\rho}^{(N)}=0, \quad \text { and } \quad \lim _{N \rightarrow \infty} \delta_{\tau}^{(N)}=0 .
$$

To prove the above Proposition 3, we make a connection with the Erlang fixed point for loss networks [6]. Specifically, we show that for the above scaling the CSMA fixed point is asymptotically identical to the Erlang fixed point of a loss network with two-hop routes where each link can support at most one connection, and use the analysis of Hajek and Krishna in [4].

\section{Achievable Rate Region}

In this section we use the CSMA fixed point approximation to characterize the achievable rate region of static CSMA schedulers. In Section VII we will show that this characterization is asymptotically accurate for large networks with a small sensing time.

Consider a wireless network with sensing time $\beta>0$ as described in Section II. Note that $\mathscr{R}$ denotes the set of routes in the network. Also, recall the definitions of $\lambda$ and $\Lambda_{i}$, $i \in \mathscr{N}$, as given in (1) and (2), respectively. Using these definitions, let the set $\Gamma(\beta)$ be given by

$\Gamma(\beta)=\left\{\lambda=\left\{\lambda_{r}\right\}_{r \in \mathscr{R}} \mid \Lambda_{i}<\tau\left(G^{+}(\beta)\right) e^{-\left(G^{+}(\beta)\right)}, i=1, \ldots, N\right\}$,

where $G^{+}(\beta)=\sqrt{2 \beta}$ (see Section III). The next proposition states that under the CSMA fixed point approximation the achievable rate region of static CSMA policies is equal to $\Gamma(\beta)$.

Proposition 4: Given $\beta>0$, for every $\lambda \in \Gamma(\beta)$ there exists a static CSMA policy p such that $\lambda_{(i, j)}<\tau_{(i, j)}(\mathbf{p})$, $(i, j) \in \mathscr{L}$.

Our proof for Proposition 4 is constructive in the sense that given a rate vector $\lambda \in \Gamma(\beta)$, we derive a static CSMA policy p such that $\lambda_{(i, j)}<\tau_{(i, j)}(\mathbf{p}),(i, j) \in \mathscr{L}$. It is also shown that the resulting policy satisfies Assumption 2, and hence is feasible.

Using the results of Section III, we have that

$$
\lim _{\beta \downarrow 0} \Gamma(\beta)=\left\{\lambda=\left\{\lambda_{r}\right\}_{r \in \mathscr{R}} \mid \Lambda_{i}<1 \quad i=1, \ldots, N\right\} .
$$

Note that any rate vector $\lambda$ for which there exists a node $i$ with $\Lambda_{i} \geq 1$ cannot be stabilized, as the service rate at each node is upper-bounded by 1 . Hence, the above result suggests that for network with a small sensing time the achievable rate region of static CSMA policies is equal to the capacity region. In the next section we show that this result is true for the limiting regime of large networks with a small sensing time.

\section{ASYMPTOTIC OPTIMALITy OF CSMA}

In this section we consider the case of large networks with a small sensing time under the many flow asymptotic.

\section{A. Many Flow Asymptotic}

Recall that in Section $\mathrm{V}$ we introduced a sequence of networks for which the number of nodes $N$ increases to infinity, and let $\mathscr{L}^{(N)}$ be the set of all links in the network with $N$ nodes, and $\mathscr{N}_{i}^{(N)}$ be the set of neighbors of node $i$ in the network with $N$ nodes. In this section, we introduce a similar scaling for the set of flows in addition to those. In particular, we let $\mathscr{R}^{(N)}$ be the set of given routes for the network with $N$ nodes and let $\lambda^{(N)}=\left\{\lambda_{r}^{(N)}\right\}_{r \in \mathscr{R}^{(N)}}$ be the mean arrival rate vector. For the mean rate vector $\lambda^{(N)}$, let

$$
\lambda_{(i, j)}^{(N)}=\sum_{r \in \mathscr{R}^{(N)}:(i, j) \in r} \lambda_{r}^{(N)}, \quad(i, j) \in \mathscr{L}^{(N)},
$$

be the total mean packet arrival rate over all routes $r \in \mathscr{R}^{(N)}$ that traverse link $(i, j)$, and let

$$
\Lambda_{i}^{(N)}=\sum_{j \in \mathscr{N}_{i}^{(N)}}\left[\lambda_{(i, j)}^{(N)}+\lambda_{(j, i)}^{(N)}\right], \quad i=1, \ldots, N
$$

be the total mean packet arrival rate over all routes $r \in \mathscr{R}^{(N)}$ that traverse node $i$.

Definition 6 (Many Flow Asymptotic): Given a sequence of networks $\left\{\mathscr{L}^{(N)}, \mathscr{R}^{(N)}\right\}_{N \geq 1}$, we define $\mathscr{D}$ as the set of all rate vector sequences $\left\{\lambda^{(N)}\right\}_{N \geq 1}$ for which the following properties hold.

(a) There exists a constant $\bar{\Lambda}$ and an integer $\bar{N}$ such that for all $N \geq \bar{N}$ we have that $\Lambda_{i}^{(N)} \leq \bar{\Lambda}, i=1, . ., N$.

(b) For every $\varepsilon>0$, there exists an integer $N_{0}$ such that for $N \geq N_{0}$ we have that $\lambda_{(i, j)}^{(N)}<\varepsilon,(i, j) \in \mathscr{L}^{(N)}$. $\diamond$ A sequence $\left\{\lambda^{(N)}\right\}_{N \geq 1} \in \mathscr{D}$ defines the limiting regime where (a) the number of nodes and routes of the network increases, (b) the arrival on each route decreases, and (c) for each node we have that the total rate over all routes that pass through the node stays bounded. The definition is closely related to the definition of large networks with diverse routing that has been used in the context of the Erlang fixed point approximation [6], [4].

We define the asymptotic achievable rate region of static CSMA policies for large networks as follows.

Definition 7: The asymptotic achievable rate region of static CSMA policies under the many flow limit is the set of sequences $\left\{\lambda^{(N)}\right\}_{N \geq 1} \in \mathscr{D}$ for which there exists a sequence of static CSMA scheduling policies $\left\{\mathbf{p}^{(N)}\right\}_{N \geq 1}$ such that

$$
\liminf _{N \rightarrow \infty}\left(\Delta_{\min }^{(N)}-1\right)>0, \quad n \geq 1 .
$$

where $\Delta_{\text {min }}^{(N)}=\min _{(i, j) \in \mathscr{L}^{(N)}} T_{(i, j)}^{(N)} / \lambda_{(i, j)}^{(N)}$.

The above definition implies that every rate sequence $\left\{\lambda^{(N)}\right\}_{N \geq 1}$ in the asymptotic rate region can eventually be stabilized by a static CSMA policy.

Note that a sequence $\left\{\lambda^{(N)}\right\}_{N \geq 1} \in \mathscr{D}$ for which there exists a node $i$ with

$$
\lim _{N \rightarrow \infty} \Lambda_{i}^{(N)} \geq 1
$$

can not be stabilized as service rate at each node is bounded by 1 . Hence, the achievable region under the many flow limit 
is contained in the set

$$
\begin{gathered}
\mathscr{C}=\left\{\left\{\lambda^{(N)}\right\}_{N \geq 1} \in \mathscr{D} \mid \exists \bar{\Lambda}<1 \text { and an integer } \bar{N}\right. \text { such that } \\
\left.\forall N \geq \bar{N} \text { we have } \Lambda_{i}^{(N)} \leq \bar{\Lambda}, i=1, \ldots, N\right\} .
\end{gathered}
$$

We refer to $\mathscr{C}$ as the capacity region under the many flow limit.

\section{B. Asymptotic Rate Region}

In this subsection we characterize the asymptotic achievable rate region of static CSMA policies under the many flow limit for networks with a small sensing time, and show that the asymptotic achievable rate region of static CSMA policies is equal to capacity region $\mathscr{C}$.

Consider the same scaling of the sensing time as in Section $\mathrm{V}$ as described in Assumption 1. We have the following result.

Proposition 5: Under the scaling $\beta^{(N)}$, for every sequence $\lambda^{(N)} \in \mathscr{C}$ there exists a sequence of static CSMA policies $\left\{\mathbf{p}^{(N)}\right\}_{N \geq 1}$ that asymptotically stabilizes the network, i.e.

$$
\liminf _{N \rightarrow \infty}\left(\Delta_{\min }^{(N)}-1\right)>0, \quad n \geq 1
$$

where

$$
\Delta_{\min }^{(N)}=\min _{(i, j) \in \mathscr{L}^{(N)}} \frac{T_{(i, j)}^{(N)}\left(\mathbf{p}^{(N)}\right)}{\lambda_{(i, j)}^{(N)}} .
$$

We provide a sketch of the proof for Proposition 5. By definition, for each sequence $\left\{\lambda^{(N)}\right\}_{N \geq 1} \in \mathscr{C}$ there exists a scalar $\bar{\Lambda}<1$ and an integer $\bar{N}$ such that for $N \geq \bar{N}$ we have

$$
\Lambda_{i}^{(N)} \leq \bar{\Lambda}, \quad i=1, \ldots, N
$$

As $\lim _{N \rightarrow \infty} \beta^{(N)}=0$ and $\lim _{\beta \downarrow 0} \tau\left(G^{+}(\beta)\right)=1$ (see Eq. (5)), there exists a integer $N_{0}$ and a constant $\varepsilon_{1}$ such that for $N \geq N_{0}$ we have that

$$
\Lambda_{i}^{(N)}<\tau\left(G^{+}\left(\beta^{(N)}\right)\right) e^{-\left(G^{+}\left(\beta^{(N)}\right)\right)}\left(1-\varepsilon_{1}\right), \quad i=1, \ldots, N .
$$

Using the proof of Proposition 4, we can then construct a sequence of static CSMA policies $\left\{\mathbf{p}^{(N)}\right\}_{N \geq 1}$ such that for $N \geq N_{0}$ we have

$$
\lambda_{(i, j)}^{(N)}<\tau_{(i, j)}\left(\mathbf{p}^{(N)}\right)\left(1-\varepsilon_{2}\right), \quad(i, j) \in \mathscr{L}^{(N)}
$$

where $\varepsilon_{2}>0$ is a constant that does not depend on $N$. Using Proposition 3, the approximation $\tau_{(i, j)}\left(\mathbf{p}^{(N)}\right)$ of the service rate of link $(i, j)$ is then asymptotically accurate as $N$ increases, and the result follows.

\section{CONCLUSIONS}

In this paper we introduced the CSMA fixed point approximation to study static CSMA schedulers in wireless networks with primary interference, and showed that the approximation is asymptotically accurate for large networks with a small sensing time. There are three important issues that are not addressed in this paper, but are being investigated in our ongoing work. First, while we showed that the CSMA fixed point approximation is asymptotically accurate, we did not investigate "how large" a network has to be in order to obtain a good approximation. We carried out several numerical case studies which suggest that the CSMA fixed point approximation is already remarkably accurate for networks where each nodes has more than 5-10 neighbours. Second, the construction of a static CSMA scheduler requires the a priori-knowledge of the arrival rate vector. We are investigating an approach using a queue-length based scheduler similar to [9] in order to obtain CSMA schedulers that are able to dynamically adapt to any rate vector in the achievable rate region. Third, the current analysis is restricted to wireless networks with primary interference, and it is an open problem to extend the analysis to more general interference models.

\section{REFERENCES}

[1] D. Bertsekas. Nonlinear Programming. Athena Scientific, Belmont, MA, 1999.

[2] D. Bertsekas and R. Gallager. Data Networks. Prentice Hall, Englewood Cliffs, NJ, 1990.

[3] L. Bui, A. Eryilmaz, R. Srikant, and X. Wu. Joint asynchronous congestion control and distributed scheduling for wireless networks. Proceedings of IEEE Infocom 2006.

[4] B. Hajek and A. Krishna. Bounds on the accuracy of the reducedload blocking formula for some simple circuit-switched networks. In Proceedings of International Conference on New Trends in Communication, Control and Signal Processing, Ankara, Turkey, 1990.

[5] S. Sarkar, and L. Tassiulas. Maxmin Fair Scheduling in Wireless Networks. In Proceedings of IEEE INFOCOM, 2002.

[6] F. P. Kelly. Loss networks. The Annals of Applied Probability, 1(3):319-378, August 1991.

[7] X. Lin and S. Rasool. Constant-time distributed scheduling policies for ad hoc wireless networks. In Proceedings of CDC, 2006.

[8] X. Lin and N. Shroff. The impact of imperfect scheduling on crosslayer rate control in multihop wireless networks. In Proceedings of IEEE Infocom, Miami, FL, March 2005.

[9] P. Marbach and Y. Lu. Active queue management and scheduling for wireless networks: The single-cell case. In Proceedings of Conference on Information Sciences and System (CISS), Princeton, NJ, 2006.

[10] G. Sharma, R. Mazumdar, and N. Shroff. On the complexity of scheduling in multihop wireless systems. In Proceedings of MobiCom, Los Angeles, CA, September 2006.

[11] L. Tassiulas and A. Ephremides. Stability properties of constrained queueing systems and scheduling policies for maximum throughput in multihop radio networks. IEEE Transactions on Automatic Control, 36:1936-1948, December 1992.

[12] X. Wu and R. Srikant. Regulated maximal matching: A distributed scheduling algorithm for multi-hop wireless networks with nodeexclusive spectrum sharing. In Proceedings of IEEE Conference on Decision and Control., 2005.

[13] M. Durvy and P. Thiran. "A Packing Approach to Compare Slotted and Non-Slotted Medium Access Control. In Proceedings of IEEE Infocom, 2006.

[14] Ch. Bordenave, D. McDonald, A. Proutiere, A Particle System in Interaction with a Rapidly Varying Envoirnment: Mean Field Limits and Applications, submitted Stochastic Processes and their Applications, 2007.

[15] D. McDonald. Elements of Applied Probability for Engineering, Mathematics, and Systems Science. World Scientific Publishing Co. Plc. Ltd., River Edge, NJ, 2003. 